\title{
Wave Forcing of Zonal Mean Angular Momentum in Various Coordinate Systems
}

\author{
JOSEPH EGGER \\ Meteorologisches Institut, Universität München, Munich, Germany \\ Klaus-Peter Hoinka \\ Institut für Physik der Atmosphäre, DLR, Oberpfaffenhofen, Germany
}

(Manuscript received 2 March 2013, in final form 19 November 2013)

\begin{abstract}
The wave forcing of the atmospheric mean flow in isentropic coordinates has been investigated intensively in the past with the divergence of the Eliassen-Palm flux playing a dominating role. These concepts are reviewed briefly and it is pointed out that angular momentum is attractive in this context because the wave driving can be written in the form of a flux divergence. This helps to evaluate the wave forcing in other coordinate systems with a different separation of waves and mean flow. The following coordinates are chosen: $(\lambda, \varphi, z),(\lambda, \varphi, \theta)$, and $(\lambda, \theta, z)$. To be consistent, only one type of zonal averaging should be used. Massweighted averaging is applied in the isentropic standard case and simple averaging is applied in the others. The wave driving is presented for all three systems. It has to balance essentially the mean-flow part of the "Coriolis term" in the angular momentum budget in $(\varphi, z)$ and $(\theta, z)$ coordinates but not in the $(\varphi, \theta)$ system where the form drag is a mean-flow term and, therefore, the forcing pattern differs from what has been published so far.
\end{abstract}

\section{Introduction}

Zonal mean momentum budgets have been long considered in order to better understand the structure and intensity of the zonal mean flow (e.g., Oort and Peixoto 1983). The contribution of the "waves" to the budgets-that is, by the deviations from the mean state-is of key interest. The so-called Eliassen-Palm (EP) flux played a dominating role in this context because its divergence "is a direct measure of the total forcing of the zonal mean state by the eddies" (Edmon et al. 1980, p. 2600), at least in the context of the transformed Eulerian-mean (TEM) equations. The EP flux divergence equals in this case the meridional potential vorticity flux in the quasigeostrophic limit and tends to be divergent near the surface in midlatitudes and convergent aloft with a subtropical extension (Edmon et al. 1980). The quasigeostrophic EP flux is

Corresponding author address: Joseph Egger, Meteorological Institute, University of Munich, Theresienstr. 37, 80333 Munich, Germany.

E-mail: j.egger@lrz.uni-muenchen.de

$$
\mathbf{F}_{g}=\left(-\overline{u^{\prime} v^{\prime}}, f^{2} N^{-2} \overline{v^{\prime} \frac{\partial \psi^{\prime}}{\partial z}}\right)
$$

in standard notation and height coordinates. The overbar stands for zonal integration and a long-term mean with primed deviations. The observed low-level divergence $\boldsymbol{\nabla} \cdot \mathbf{F}_{g}$ is closely related to the meridional eddy temperature transport while the upper-level patterns reflect mainly the eddy momentum flux. Thus, (1.1) provides a clear formulation of wave forcing in the TEM equations. Of course, (1.1) is not the only possibility to quantify a wave forcing. We may switch from the TEM equations to the standard equations for momentum or angular momentum. The height coordinates in (1.1) may be replaced by other coordinates and we may apply a mass-weighted averaging instead of the simple zonal averaging in (1.1). The last point is surprisingly important, as will be demonstrated by turning to isentropic coordinates. Following Andrews (1983), we start from the equation of zonal momentum in $f$-plane notation [Andrews 1983, his (2.14)] 


$$
\begin{gathered}
\frac{\partial}{\partial t}(u \sigma)+\frac{\partial}{\partial x}\left(u^{2} \sigma\right)+\frac{\partial}{\partial y}(u v \sigma) \\
+\frac{\partial}{\partial \theta}(\dot{\theta} u \sigma)-f v \sigma+\sigma \frac{\partial M}{\partial x}=0,
\end{gathered}
$$

with $\sigma=-g^{-1}(\partial p / \partial \theta)$, Montgomery potential $M$, horizontal velocity $(u, v)$, and heating $\dot{\theta}$. When deriving the wave forcing it is standard to treat the term

$$
\sigma \frac{\partial M}{\partial x}=\sigma f v_{g}
$$

with geostrophic meridional wind $v_{g}$, differently from the Coriolis term in (1.2) although both terms are clearly of the same type. Zonal averaging is applied to the Montgomery term, which is then transformed into the derivative of part of the vertical component of $\mathbf{F}$ at least if $\overline{v_{g}}=0$ while the Coriolis term is exposed to the massweighted average

$$
\tilde{a}=\overline{a \sigma} \bar{\sigma}^{-1}
$$

The result is

$$
\mathbf{F}=\left(-\overline{(\sigma v)^{\prime} u^{\prime}}, \overline{(\sigma u)^{\prime} \dot{\theta}^{\prime}}+\overline{p^{\prime}\left(\frac{\partial M}{\partial x}\right)^{\prime}}\right)
$$

for the EP flux vector where steady state is assumed. It is assumed in (1.5) that the isentropic surfaces do not intersect the ground. This assumption excludes the deep surface zone (Koh and Plumb 2004) but Andrews (1983) derived also a flux formula for this more complicated situation.

This procedure is formally correct, of course, but the Montgomery term is then counted as wave forcing (form drag) while $f \tilde{v} \bar{\sigma}$ is seen as a mean-flow term. If, however, both terms are treated equally, when averaging, the Coriolis term becomes

$$
f \overline{v \sigma}=f\left(\bar{v} \bar{\sigma}+\overline{v^{\prime} \sigma^{\prime}}\right)
$$

and the geostrophic part $f \overline{\left(v_{g}^{\prime} \sigma^{\prime}\right)}$ of the eddy term cancels the form drag. The remaining term $\overline{f v_{\mathrm{ag}}^{\prime} \sigma^{\prime}}$, with ageostrophic wind component $v_{\text {ag }}$, is a wave forcing but cannot be written as a divergence.

We may just as well apply mass-weighted averaging as consistently as possible. The Montgomery term becomes a mean-flow term in that case and we obtain, with

$$
f \tilde{v}-\frac{\partial \tilde{M}}{\partial x}=f \tilde{v}_{\mathrm{ag}},
$$

a flux as in (1.5), but without the second term of the vertical component.
It is one of our main points that only one type of averaging should be used. As far as we can see, all evaluations of wave forcing in isentropic coordinates published as yet mix both types of averaging as in Andrews (1983). Thus, the averaging conventions strongly affect both form and meaning of the wave forcing. On the other hand, there is no difference between these averaging procedures in pressure coordinates.

As pointed out by Tung (1986), it is conceptually simpler to consider angular momentum (AM) instead of zonal momentum because AM satisfies a conservation equation so that, for example, wave driving becomes a flux divergence, at least if there are no intersections. As stated above, the structure of mean flow and eddies reflects the choice of the coordinate system. Thus, AMbased evaluations of the wave forcing in isentropic coordinates (e.g., Iwasaki 1992; Tanaka et al. 2004; Koh and Plumb 2004) should be complemented by work in height coordinates or other systems that are useful for general circulation studies. For example, Yang et al. (1990) evaluated the wave driving in terrain-following coordinates in the troposphere. This choice was motivated by the wish to avoid the intersection problem.

Three coordinate systems will be considered below. Height coordinates $(\lambda, \varphi, z)$ are selected as being the simplest and most obvious choice. As stated above, the isentropic coordinates $(\lambda, \varphi, \theta)$ played a key role in the development of EP flux concepts and will be, of course, included here as well. Finally, an example with an unusual meridional coordinate-namely $(\lambda, \theta, z)$-will be presented to widen the perspective of the discussion. Nurser and Lee (2004) and Nycander et al. (2007) used density as a meridional coordinate in the ocean. Pauluis and Mrowiec (2013) used equivalent potential temperature $\theta_{e}$ as a coordinate in $\left(\theta_{e}, z\right)$ space in order to analyze convective motions. The $(\theta, z)$ system appears to have the same advantages as the conventional isentropic coordinates but avoids the intersection problem at the surface. Contortions of isotherms create difficulties, however.

The AM budget equation and the definition of wave driving will be given in section 2 in general coordinates. Results are presented in section 3.

\section{Angular momentum budgets in general coordinate systems}

Let us formulate the problem in a general $\left(\lambda, q_{2}, q_{3}\right)$ system where

$$
J=a^{2} \cos \varphi\left(\frac{\partial \varphi}{\partial q_{2}} \frac{\partial z}{\partial q_{3}}-\frac{\partial \varphi}{\partial q_{3}} \frac{\partial z}{\partial q_{2}}\right)
$$

is the geometric transformation factor. The equation of continuity is 
$\frac{\partial}{\partial t}(\rho J)+\frac{\partial}{\partial \lambda}(\rho J \dot{\lambda})+\frac{\partial}{\partial q_{2}}\left(\rho J \dot{q}_{2}\right)+\frac{\partial}{\partial q_{3}}\left(\rho J \dot{q}_{3}\right)=0$

and angular momentum conservation reads

$$
\begin{aligned}
\frac{\partial}{\partial t} & (m J)+\frac{\partial}{\partial \lambda}(\dot{\lambda} m J)+\frac{\partial}{\partial q_{2}}\left(\dot{q}_{2} m J\right)+\frac{\partial}{\partial q_{3}}\left(\dot{q}_{3} m J\right) \\
& =-\left.\frac{\partial p}{\partial \lambda}\right|_{z, \varphi} J+R
\end{aligned}
$$

with angular momentum per unit volume $m=\rho(u+$ $\Omega a \cos \varphi) a \cos \varphi$. The rhs pressure gradient will be adapted to the specific coordinate systems below. The "friction" term $R$ is an eddy term. AM conservation implies that both rhs terms can be written as a divergence.

The separation of wave and mean flow is problematic in the surface zone in $(\lambda, \varphi, \theta)$ coordinates, which contains all those isentropic surfaces that sometimes or always intersect the ground (Koh and Plumb 2004). Of course, $\overline{v_{g}}=0$ above this zone [see (1.3)] but let us consider the situation when a certain isentrope outcrops just in the interval $\lambda_{1}<\lambda<\lambda_{2}$. We have to define zonal average procedures adapted to this intersection problem. It is standard practice to assume a vanishing density outside the interval $\lambda_{1}<\lambda<\lambda_{2}$ (e.g., Lorenz 1955) when averaging zonally but we follow here Koh and Plumb (2004), who considered only isentropes above the ground. In what follows, the overbar means as before

$$
\bar{b}=(2 \pi)^{-1} \int_{\lambda_{1}}^{\lambda_{2}} b d \lambda,
$$

while

$$
\hat{b}=\left(\lambda_{2}-\lambda_{1}\right)^{-1} \int_{\lambda_{1}}^{\lambda_{2}} b d \lambda
$$

is the zonal mean of a variable $b$ with respect to the interval with perturbation $b^{\prime}=b-\hat{b}$. Of course, $\bar{b}=$ $\hat{b}\left(\lambda_{2}-\lambda_{1}\right)(2 \pi)^{-1}$ with $\lambda_{1}=0$ and $\lambda_{2}=2 \pi$ if there are no intersections. Many intersections are treated correspondingly. Using this notation, we average (2.3) over longitude and time and collect all eddy terms on the rhs so that

$$
\begin{aligned}
\frac{\partial}{\partial q_{2}}\left(\overline{\dot{q}}_{2} \hat{J} \hat{m}\right)+\frac{\partial}{\partial q_{3}}\left(\overline{\dot{q}}_{3} \hat{J} \hat{m}\right) \\
=-\frac{\partial}{\partial q_{2}}\left(\overline{\dot{q}}_{2} J m-\overline{\dot{q}}_{2} \hat{J} \hat{m}\right) \\
\quad-\frac{\partial}{\partial q_{3}}\left(\overline{\dot{q}}_{3} J m-\overline{\dot{q}}_{3} \hat{J} \hat{m}\right)-\frac{\overline{\partial p}}{\partial \lambda}+\bar{R} .
\end{aligned}
$$

It has been shown by Andrews (1983) for the isentropic system that zonal averaging commutes with the differential operators in (2.6) even in the presence of intersections. A similar proof can be given in the general case, at least if $J \neq 0$. However, specific problems come up in $(\theta, z)$ coordinates as will be discussed below.

The transformation to $\left(\lambda, p_{2}, q_{3}\right)$ coordinates is possible only if $J$ is finite and has the same sign throughout the flow domain. Neither the $(\varphi, \theta)$ system nor the $(\theta, z)$ system satisfy this condition. It is standard custom to neglect this point in $(\varphi, \theta)$ coordinates where unstable or neutral stratification is almost restricted to the boundary layer. However, points with $\partial \theta / \partial \varphi \geq 0$ can be found everywhere in the Northern Hemisphere. Thus, (1.2) and (1.3) are not directly applicable, nor can (2.4) be evaluated on an isentrope. Pauluis and Mrowiec (2013) solved this problem with respect to mass conservation. Using similar methods it will be shown in the appendix how to cast (2.6) in a form that is compatible with the $(\theta, z)$ system.

We follow Tung (1986) and Yang et al. (1990) by equating $\boldsymbol{\nabla} \cdot \mathbf{F}$ to the divergence of AM mean fluxes, so that the wave forcing

$$
\boldsymbol{\nabla} \cdot \mathbf{F}=\frac{\partial}{\partial q_{2}}\left(\overline{\dot{q}}_{2} \hat{m} \hat{J}\right)+\frac{\partial}{\partial q_{3}}\left(\overline{\dot{q}}_{3} \hat{m} \hat{J}\right)
$$

balances the lhs of (2.6) where $\boldsymbol{\nabla} \cdot \mathbf{F}$ denotes henceforth the forcing in general. The wave driving is completely due to eddy action and includes also turbulent subgrid transfers. Its impact in the AM budget must be balanced by the divergence of mean AM fluxes. The formulation (2.7) has the advantage that only zonal-mean-flow variables must be evaluated. Zonal averaging in the case of intersecting coordinate surfaces enforces, however, modifications to be discussed below.

Data will be used to evaluate the mean-flow divergence in (2.7), which has to balance all "wave effects" including friction terms. The evaluation of the divergences is based on the 40-yr European Centre for Medium-Range Weather Forecasts (ECMWF) Re-Analysis (ERA-40) set for the years 1958-2001, which contains winds, temperature, pressure, and also $\dot{T}$ as caused by "physics." The calculations are performed for December-February (DJF). The horizontal resolution of the data is $2.25^{\circ} \times$ $2.25^{\circ}$. The time resolution is $6 \mathrm{~h}$. Vertical interpolation creates the values on height surfaces and isentropes when needed. The vertical resolution is $D z=1000 \mathrm{~m}$ in $(\varphi, z)$, $D z=500 \mathrm{~m}$ in $(\theta, z)$, and $D \theta=3 \mathrm{~K}$ in $(\varphi, \theta)$ coordinates. It is convenient to display normalized flux divergences $J_{N}^{-1} \nabla \cdot \mathbf{F}$, where $J_{N}$ is a suitably chosen mean value of $J$. This normalization ensures that the dimension of all flux divergences is kilograms per meter per squared second. 
It is partly a matter of taste if one accepts $m$ as a basic variable so that $\bar{m}$ is a mean-flow variable. This is the view chosen here except for isentropic coordinates where the expression $\rho J=-g^{-1}(\partial p / \partial \theta) a^{2} \cos \varphi$ [see (2.1)] has such a convenient form that $m^{*}=m \rho^{-1}$ is selected as basic variable.

\section{Results}

In what follows, the wave forcing will be presented in $\left(q_{2}, q_{3}\right)$ planes according to (2.7). One expects that the mean "Coriolis term"

$C=-2 \Omega a^{2} \cos \varphi \sin \varphi \hat{J} \hat{\rho}\left(\overline{\dot{q}}_{2} \frac{\widehat{\partial \varphi}}{\partial q_{2}}+\overline{\dot{q}}_{3} \frac{\widehat{\partial \varphi}}{\partial q_{3}}\right) \sim \nabla \cdot \mathbf{F}$

is the dominant rhs term in (2.7) at least off the equator.

\section{a. $(\varphi, z)$ coordinates}

Orography intersects constant-height surfaces. That causes problems because the local contributions to friction and mountain torques vary with height. However, wave forcing is so noisy near the surface that such effects cannot be properly dealt with here. With $J_{N}=$ $a^{2} \cos \varphi,(2.7)$ becomes

$J_{N}^{-1} \nabla \cdot \mathbf{F}=(a \cos \varphi)^{-1} \frac{\partial}{\partial \varphi}(\bar{v} \hat{m} \cos \varphi)+\frac{\partial}{\partial z}(\bar{w} \hat{m})$

and (3.1) yields

$$
J_{N}^{-1} \nabla \cdot \mathbf{F} \approx-\hat{\rho} \bar{v}_{\mathrm{ag}} 2 \Omega a \cos \varphi \sin \varphi .
$$

Poleward (equatorward) flow implies negative (positive) wave forcing. The flux divergence in Fig. 1a exhibits relatively clear structures in the upper troposphere and lower stratosphere. Noise is the dominant feature in the lower troposphere. Regions with grid points inside the orography have been blackened. This procedure excludes rather noisy domains. Maximum values are approximately $10^{2} \mathrm{~kg} \mathrm{~m} \mathrm{~s}^{-2}$. This corresponds with a tendency $\partial \bar{u} / \partial t \sim 2 \mathrm{~m} \mathrm{~s}^{-1}$ day $^{-1}$ near $z=0$.

The noisiness of Fig. 1a suggests deletion of the massflow convergence terms in (2.7) and to use the approximate form

$$
J_{N}^{-1} \boldsymbol{\nabla} \cdot \mathbf{F} \sim \hat{\rho}\left(\bar{v} a^{-1} \frac{\partial}{\partial \varphi} \hat{m}^{*}+\bar{w} \frac{\partial}{\partial z} \hat{m}^{*}\right),
$$

which is a slightly improved version of (3.1) with $m^{*}=$ $m \rho^{-1}$. The result is displayed in Fig. 1b. Obviously (3.4) provides a smooth approximation to Fig. 1a so that the

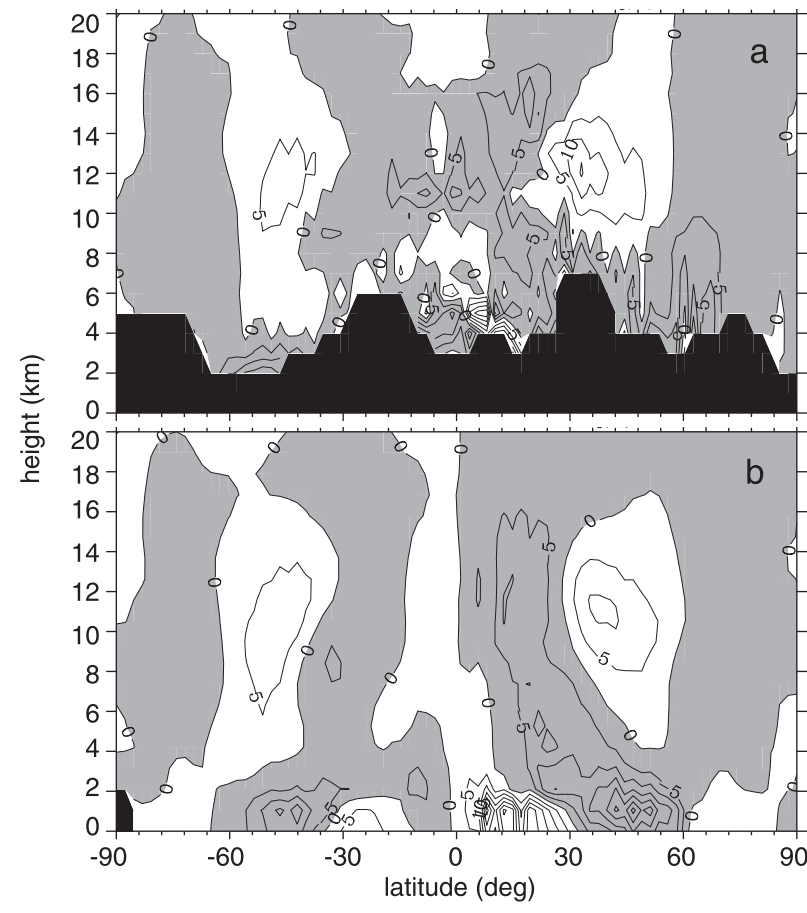

FIG. 1. Normalized wave forcing $(2.7)$ in $(\varphi, z)$ coordinates $\left(10^{1} \mathrm{~kg} \mathrm{~m} \mathrm{~s}^{-2}\right)$ in height coordinates for DJF. (a) Full representation (3.2) but with blackening of mountain grid points as explained in the text; (b) approximate form (3.4); $J_{N}=a^{2} \cos \varphi$.

lowest parts of the troposphere can be included as well. The only major difference is found near the equator where (3.4) indicates divergence throughout the depth of the atmosphere while Fig. 1a indicates mainly convergence for $z<15 \mathrm{~km}$. Figure 1 supports the estimate (3.3). In particular, the Ferrel cells are clearly represented in both hemispheres as is the circulation of the tropical Hadley cell in Fig. 1b.

The wave forcing in Fig. 1 differs profoundly from what has been found in isentropic coordinates. The wave driving would not converge to the potential vorticity flux in the quasigeostrophic limit. Mixing of zonal and massweighted averaging would not lead to strongly different results in $(\varphi, z)$ coordinates because there is no form drag and eddy mass fluxes are small (Egger and Hoinka 2011).

\section{b. $(\varphi, \theta)$ coordinates}

For reasons to be explained below, mass-weighted averaging had to be chosen in standard isentropic coordinates. The Montgomery term [see also (1.2)] becomes, with

$$
\rho J=-g^{-1} a^{2} \cos \varphi \frac{\partial p}{\partial \theta}
$$


and the mean flow term,

$$
T_{0}=g^{-1} a^{2} \cos \varphi \frac{\widetilde{\partial M}}{\partial \lambda} \frac{\widehat{\partial p}}{\partial \theta} .
$$

It is standard to convert this term to a vertical derivative if there are no intersections (e.g., Andrews 1983). However, we obtain, with intersections,

$$
\begin{aligned}
\overline{\frac{\partial M}{\partial \lambda} \frac{\partial p}{\partial \theta}=} & \frac{\partial}{\partial \theta}\left(\overline{p \frac{\partial M}{\partial \lambda}}\right)-\left.\left(\frac{\partial M}{\partial \lambda} p \frac{\partial \lambda}{\partial \theta}\right)\right|_{\lambda_{1}} ^{\lambda_{2}} \\
& -\left.R c_{p}\left(R+c_{p}\right)^{-1} p_{0}^{-R / c_{p}}\left(p^{\left(R+c_{p}\right) / c_{p}}\right)\right|_{\lambda_{1}} ^{\lambda_{2}} .
\end{aligned}
$$

The wave driving cannot be written as a flux divergence (see also Koh and Plumb 2004) if there are intersections. The mean-flow term $T_{0}$ must be transferred to the lhs of (2.6). Thus, the wave forcing is

$$
\begin{aligned}
(\boldsymbol{\nabla} \cdot \mathbf{F})^{+}= & \frac{\partial}{\partial \varphi}\left(-g^{-1} \widehat{m^{*}} \frac{\widehat{\partial p}}{\partial \theta} \tilde{v} a \cos \varphi\right) \\
& +\frac{\partial}{\partial \theta}\left(-g^{-1} \widetilde{\dot{\theta}} \widehat{m^{*}} \frac{\widehat{\partial p}}{\partial \theta} a^{2} \cos \varphi\right) \\
& -g^{-1} a^{2} \cos \varphi \frac{\widetilde{\partial M}}{\partial \lambda} \frac{\widehat{\partial p}}{\partial \theta}
\end{aligned}
$$

where the symbol $(\nabla)^{+}$indicates that the rhs term in (3.8) is not a divergence. The approximation (3.1) is not useful in this case because the term $-T_{0}$ in (3.8) is important. Difficulties with the evaluation of zonally averaged mean fluxes led us to adopt mass-weighted averaging. The ERA set offers values of temperature tendency owing to physics that allow one to calculate $\dot{\theta}$. The resulting values of $\overline{\dot{\theta}}$ look rather reasonable but both $\partial \bar{v} / \partial \varphi$ and $\overline{\partial \dot{\theta}} / \partial \theta$ are found to be negative close to the ground. The mean mass flux is, therefore, extremely convergent near the surface. Although that is possible, such a feature is rather doubtful. It is likely that this problem has to do with the intersection of $\theta$ surfaces with the ground. The only solution accessible to us was to compute via

$$
\frac{\partial}{\partial \theta}\left(\overline{\dot{\theta} \frac{\partial p}{\partial \theta}}\right)=-(a \cos \varphi)^{-1} \frac{\partial}{\partial \varphi}\left(\overline{v \frac{\partial p}{\partial \theta}} \cos \varphi\right)
$$

the "vertical density flux" from the known horizontal flux, which can be evaluated directly. This procedure replaces the products $\bar{v} \widehat{\partial p / \partial \theta}$ and $\overline{\dot{\theta}} \overline{\partial p / \partial \theta}$ of the mean factors in (3.8) by the mean values of the products. It is demonstrated in Fig. 2 that these changes are not negligible. The term $\bar{v} \overline{\partial p / \partial \theta}$ in Fig. 2a is smaller than $\overline{v \partial p / \partial \theta}$

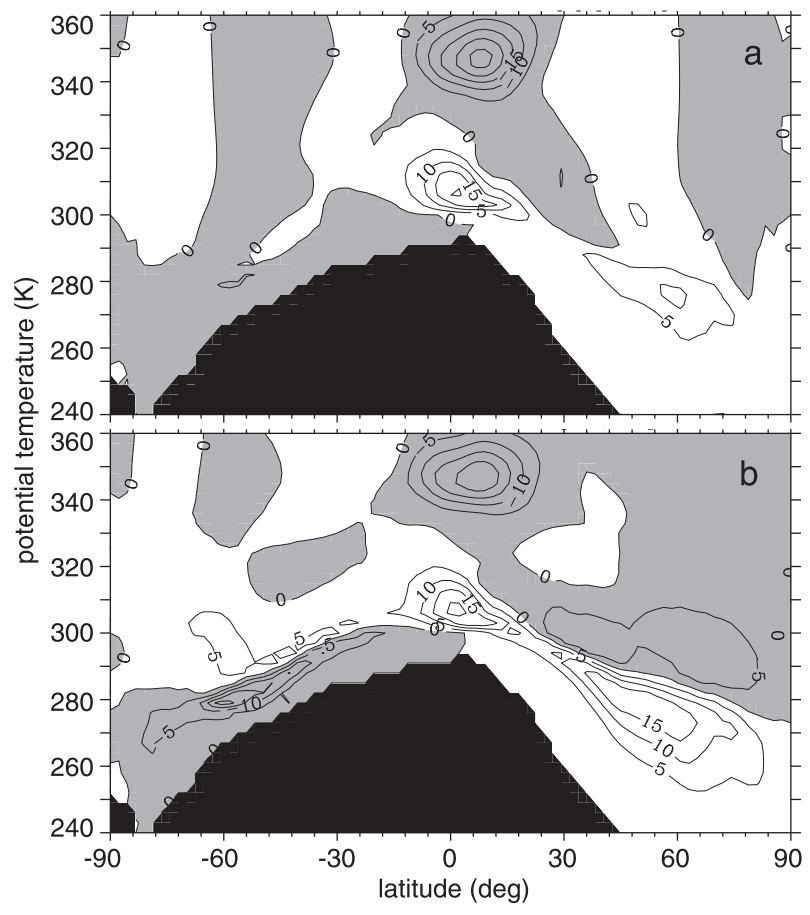

FIG. 2. Intercomparison of meridional "mass fluxes" $\left(10^{2} \mathrm{~kg} \mathrm{~K}^{-1} \mathrm{~s}^{-3}\right)$ for DJF. (a) $\bar{v} \widehat{\partial p / \partial \theta}$; (b) $\overline{v \partial p / \partial \theta}$.

in Fig. $2 b$ in most of the surface zone. Moreover, the patterns disagree completely for $\theta>290 \mathrm{~K}$ in midlatitudes. The total mass flux is directed poleward in the upper branch of the hemispheric "Hadley circulation" right from the equator to the pole (see Fig. 2b). In contrast, the meridional velocities are directed equatorward above $\theta \sim 290 \mathrm{~K}$ for $30^{\circ} \leq|\varphi| \leq 70^{\circ}$ (Fig. 2a).

Figure 2 illustrates our remarks with respect to (1.3) and (1.4). The density-weighted average in Fig. 2b contains important contributions by eddies. To accept $\tilde{v}$ as mean flow implies taking important effects out of wave forcing.

The term $(\boldsymbol{\nabla} \cdot \mathbf{F})^{+}[$see (3.8)] is displayed in Fig. 3a. In particular, intersections are fully incorporated. The results of Iwasaki (1992), Yang et al. (1990), and Tanaka et al. (2004) are based on hybrid coordinate systems that circumvent the intersection problem. They all found divergence in the lower troposphere and convergence above. Divergences are seen in Fig. 3 a near the lower boundary and higher up near the equator and at midlatitudes. Convergence is dominant in most of the northern troposphere. In particular, the zone of intense divergence near the surface, as found, for example, by Tanaka et al. (2004) is missing in Fig. 3a. The deep zone of divergence in the southern midlatitudes in Fig. 3a is new. All these differences are due to the consistent mass-weighted averaging, which treats the Montgomery 


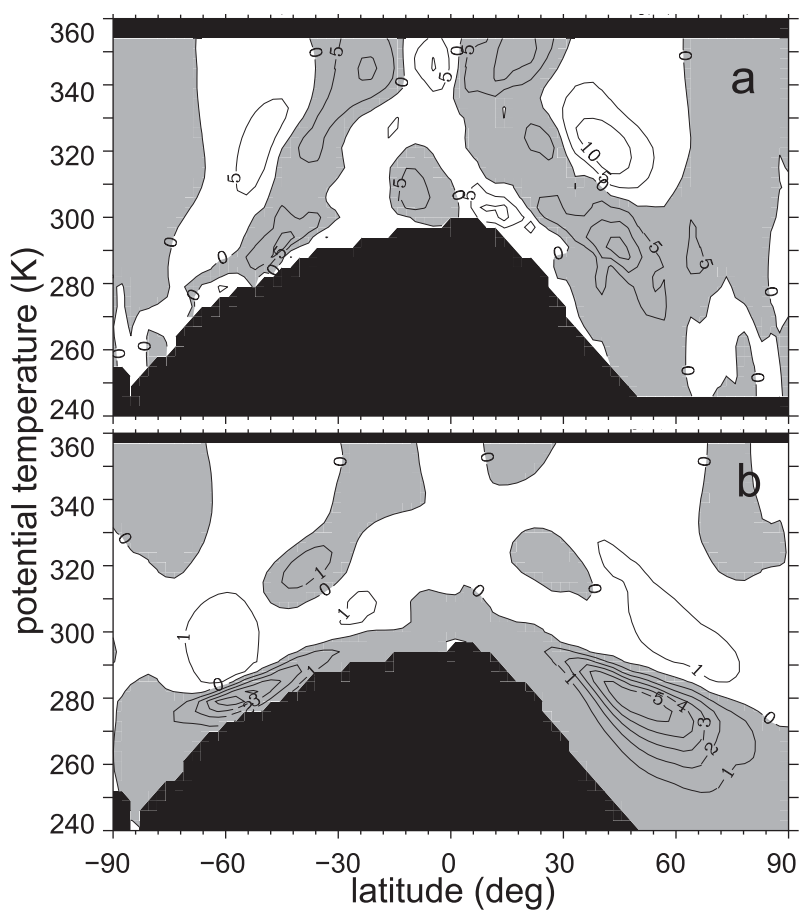

FIG. 3. Normalized wave forcing $(2.7)$ in $(\varphi, \theta)$ coordinates (DJF). (a) $J_{N}^{-1} \cdot(\boldsymbol{\nabla} \cdot \mathbf{F})^{+}\left(10^{1} \mathrm{~kg} \mathrm{~m} \mathrm{~s}^{-2}\right)$ according to (3.8) but with mass-weighted velocities $\tilde{v}, \tilde{\theta}$ [see (1.4)]. (b) $J_{N}^{-1} \cdot\left(-T_{0}\right)\left[10^{2} \mathrm{~kg} \mathrm{~m} \mathrm{~s}^{-2}\right.$; see (3.6)]. Normalization factor $J_{N}=6.9 \times 10^{15} \cos \varphi\left(\mathrm{m}^{2} \mathrm{~K}^{-1}\right)$.

term as a mean-flow term. It is, however, of interest that the "form drag" $T_{0}$ contains a substantial part due to the mean geostrophic wind $\bar{v}_{g}$ in the surface zone (not shown). This part is quite similar to the full term $T_{0}$ displayed in Fig. $3 \mathrm{~b}$. The term $-T_{0}$ is substantially larger than the divergences and convergences in Fig. 3a (note the different scalings in Figs. 3a and 3b). This means that substantial cancellation occurs in Fig. 3a. The quasigeostrophic wave forcing is at best a crude approximation to Fig. 3. The large deviations of our results from earlier ones illustrate vividly the importance of a consistent averaging procedure.

\section{c. $(\theta, z)$ coordinates}

As mentioned above, coordinates similar to $(\theta, z)$ coordinates have been used by Nurser and Lee (2004) and Nycander et al. (2007) in the ocean. Such coordinates are problematic in the atmosphere because isentropes can be quite convoluted and closed isotherms may form in a constant-height plane. The percentage of points along a latitude circle with $\partial \varphi / \partial \theta<0$ has been evaluated in the Northern Hemisphere. The percentage amounts to about $90 \%$ in midlatitudes but drops to about $60 \%$ near the equator. As stated above, a coordinate transformation as assumed in (2.1) is not possible. The situation changes, however, with zonal averaging because we can then apply an area-integrating technique developed by Butchart and Remsberg (1986), Nakamura $(1995,1996)$, and others. Our approach is described in the appendix and leads to a finite difference version of (2.6) and (2.7) but with a redefined averaging operator.

The flow domain in these coordinates spans only the lowest $10 \mathrm{~km}$ of the atmosphere because the mean meridional gradient of $\theta$ changes sign above that height. The domain slants equatorward with height (see Fig. 4). The black boundaries in Fig. 4 extending from 300 to $340 \mathrm{~K}$ represent approximately the equator. All boundaries are posed by definition except for the lower one. In any case, the "intersection problem" is hardly relevant in $(\lambda, \theta, z)$ coordinates.

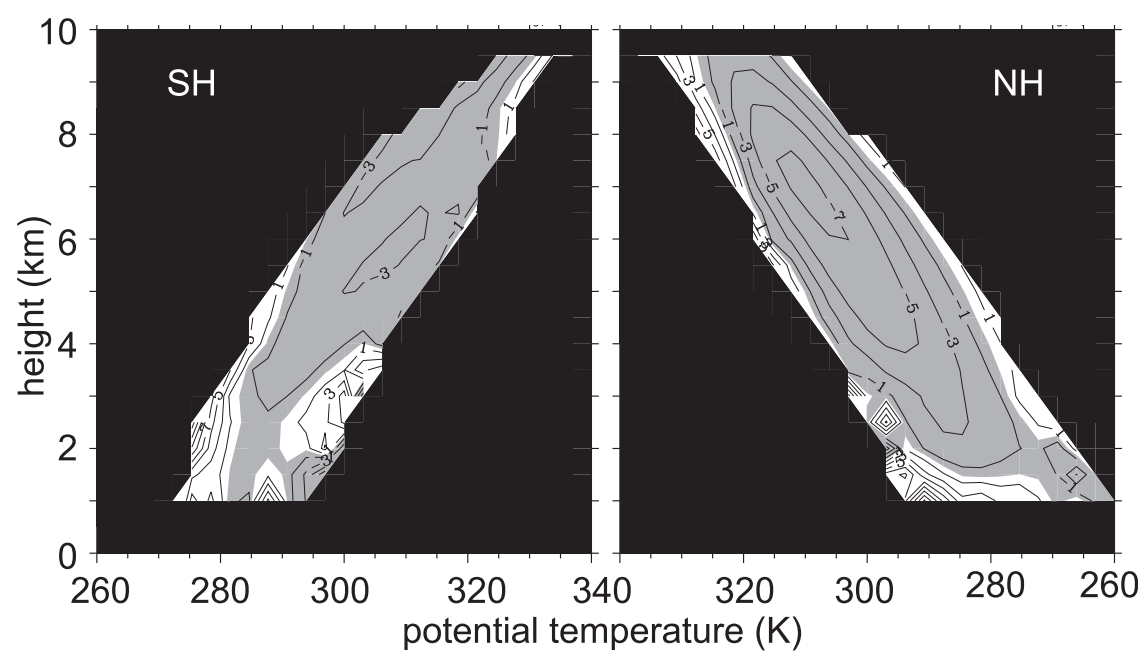

FIG. 4. Normalized wave forcing $(2.7)$ in $(\theta, z)$ coordinates $\left(10^{2} \mathrm{~kg} \mathrm{~m} \mathrm{~s}^{-2}\right) ; J_{N}$ equals the long-term mean of $J$. 
The mean mass circulation is similar to that in $(\lambda, \varphi, \theta)$ coordinates with a grand hemispheric cell (not shown). This pattern is relatively easy to understand because "meridional" motion in these coordinates is proportional to $\dot{\theta}$ with equatorward flow near the ground and in the ascending branch of the Hadley cell. There is flow through the equatorial and the upper boundary. It is a weakness of $(\theta, z)$ coordinates that $\partial \theta / \partial \varphi \sim 0$ close to the equator. Resolution is therefore rather coarse there. The normalization factor $J_{N}$ is chosen to equal the mean area of a stripe. This choice guarantees that all stripes have the same weight in Fig. 4 but leads in turn to relatively large values of the normalized forcing.

The wave forcing adds AM near the ground and near the equator in the Northern Hemisphere and removes it elsewhere. There is moderate similarity with Fig. 3a. Averaging along isentropes at constant height is obviously not the same as at constant latitude, particularly in the surface zone. The hemispheres are similar. The estimate (3.1) assumes an admissible coordinate transformation and cannot be applied here. Nevertheless, equatorward (poleward) flow goes essentially with positive (negative) forcing.

\section{Discussion}

This work is concerned with a modification and extension of the wave-forcing evaluations in the literature. The evaluation of the fluxes from data has a long tradition (see Johnson 1989 and references therein). Following Tung (1986), the forcing has been calculated for AM because it can be written as a divergence in this case. It has been stressed that zonal averaging has to be performed as consistently as possible. Otherwise, dynamically similar terms are counted sometimes as mean flow terms but sometimes as wave forcing. This requirement favors simple zonal averaging as has been applied in $(\varphi$, $z)$ and $(\theta, z)$ coordinates. Mass-weighted averaging has been used in $(\theta, z)$ coordinates.

Let us turn first to the results in $(\lambda, \varphi, \theta)$ coordinates that attracted most of flux research. Iwasaki $(1988,1992)$ used both averaging procedures in his wave-forcing calculations as did Yang et al. (1990) and Tanaka et al. (2004). They all obtained divergence (convergence) near the surface (aloft) throughout the northern troposphere in agreement with (3.1). When evaluating the flux divergence according to (2.7), unexpected accuracy problems forced us to turn to mass-weighted averages where the form drag becomes a mean-flow term. The wave driving with mass-weighted averaging deviates far from the standard results of Yang et al. (1990) and Tanaka et al. (2004). There is "convergence" in most of the Northern Hemisphere (Fig. 3a). The wave motion decelerates the mean AM in most of the lower parts of the surface zone. The estimate (3.1) for the wave forcing [see also (3.4)] is satisfied quite well (see Fig. 1). The wave forcing in spherical coordinates supports the estimate (3.3). The Coriolis term balances the forcing.

The wave driving in $(\lambda, \theta, z)$ coordinates has the form of a divergence if we restrict the analysis to isentropic latitudinal boundaries. Given $\overline{\dot{\theta}}$, it is then relatively easy to estimate the mean vertical motion and the wave driving. Equatorward motion implies divergence, and poleward motion implies convergence.

The discussion with respect to averaging shows that the concept of wave driving is not as clear cut as one might wish. The structure of the wave part depends not only on the coordinate system but also on the averaging methods. Moreover, the separation of waves and mean flow is not always obvious as is the case, for example, in the surface zone for $(\varphi, \theta)$ coordinates. These points are illustrated in this paper.

Relatively little has been said about the role of friction processes. We took here the view that friction is closely related to turbulent motion and is, therefore, part of the wave forcing. As such, friction is part of $\boldsymbol{\nabla} \cdot \mathbf{F}$. This view is convenient because it saves us from evaluating terms that are not available in the ERA datasets. Moreover, friction is generally thought to be small in the free atmosphere. There is, however, the problem that the surface zone in $(\varphi, \theta)$ coordinates is deep, so that transfer of AM between the atmosphere and Earth must be assumed throughout this zone. A close look at this problem reveals that this transfer cannot be written as a divergence. It is not captured by (2.7) and deserves future attention.

In the past, flux investigations where closely related to nonacceleration theorems (e.g., Andrews and McIntyre 1976) where conditions are explored that lead to vanishing wave forcing. Such conditions are, of course, not found in the global climate datasets used here. The atmosphere is always in such a state that wave forcing is important. No coordinate system will lead to a climatic state with $\boldsymbol{\nabla} \cdot \mathbf{F}=0$.

All in all, our results show that there is a great variability with respect to the wave forcing patterns. We may anticipate further variations when considering, for example, isertelic coordinates (Juckes 2001).

Acknowledgments. We thank both reviewers for their detailed and helpful comments.

\section{APPENDIX}

\section{Zonally Averaged AM Equation in $(\theta, z)$ Coordinates}

The AM equation in spherical height coordinates is 


$$
\begin{gathered}
\frac{\partial}{\partial t} m+(a \cos \varphi)^{-1}\left[\frac{\partial}{\partial \lambda}(u m)+\frac{\partial}{\partial \varphi}(v m \cos \varphi)\right] \\
+\frac{\partial}{\partial z}(w m)=-\frac{\partial p}{\partial \lambda}
\end{gathered}
$$

with $J=a^{2} \cos \varphi$ [see (2.3)] where friction is omitted. Let us define $\theta$ tubes that cover the space between two isentropic surfaces with $\theta=\theta_{1}$ and $\theta_{2}\left(D \theta=\left|\theta_{1}-\theta_{2}\right|\right)$ and between two heights $z_{1}$ and $z_{2}$ at distance $D z$. Integration of (A.1) over the corresponding volume $V$ yields

$$
\int_{V} \frac{\partial}{\partial t} m d V+\int_{F}(\mathbf{j} \cdot \mathbf{n}) d f=-\int_{F} p\left(\mathbf{e}_{1} \cdot \mathbf{n}\right) d f
$$

where $\mathbf{j}$ is the advective AM flux, $\mathrm{F}$ the surface of $V$ with normal vector $\mathbf{n}$ pointing outward, and $\mathbf{e}_{1}$ is the zonal unit vector. Of course, $\mathbf{n}$ equals the vertical unit vector on height surfaces and $\mathbf{n}=\boldsymbol{\nabla} \theta /|\mathbf{\nabla} \theta|$ on isentropes. It is helpful to express $\mathbf{j}$ in terms of the covariant basic vectors of the $(\theta, z)$ system [see Zdunkowski and Bott (2003) for details]. This has the advantage that two of the three basic vectors at a point are embedded in the isentropic surface on which this point is located. The corresponding flux components do not contribute to the isentropic part of the surface integral in (A.2). The only remaining vector is

$$
\mathbf{j}_{2}=\left(\dot{\theta} \frac{\partial y}{\partial \theta}+\frac{\partial y}{\partial t}\right) \mathbf{e}_{2}
$$

with unit vector $\mathbf{e}_{\mathbf{2}}$ in the meridional direction and meridional isentrope position $y(\lambda, \theta, z, t)$. Thus, $(\mathbf{j} \cdot \mathbf{n})=$ $m \dot{\theta}|\nabla \theta|^{-1}$ is finite even if $\partial y / \partial \theta$ is infinite and (A.2) becomes

$$
\begin{aligned}
& \frac{d}{d t} \int_{V} m d V-\left.\int_{F_{\theta}} m \dot{\theta}|\nabla \theta|^{-1} d f_{\theta}\right|_{\theta_{1}} ^{\theta_{2}}+\left.\int_{F_{z}} w m d f_{z}\right|_{z_{1}} ^{z_{2}} \\
& \quad=-\left.\int p \frac{\partial \theta}{\partial x}|\nabla \theta|^{-1} d f_{\theta}\right|_{\theta_{1}} ^{\theta_{2}}
\end{aligned}
$$

where $d f_{\theta}$ is an area element of a bounding $\theta$ surface and $d f_{z}$ is an element of the bounding height surfaces. The rhs term in (A.4) is the pressure torque acting on the isentropes. It is easy to show that the time derivative in (A.3) helps to move the time derivative in (A.2) out of the integral. Thus, (A.4) is the zonal mean AM equation without singularities needed for our analysis. We do not have to discuss its implementation in the analysis grid because (2.7) requires only the evaluation of some mean values where we use the approximation

$$
\bar{b}=\sum b_{i k} F_{i k} / \sum F_{i k},
$$

where $F_{i k}$ is the area of a grid box of the analysis grid at level $z_{1}$, say, and where the sum runs over all indices $(i, k)$ of the boxes of a tube at that level. In particular, $\bar{J}$ represents the area of the tube divided by $D \theta$.

\section{REFERENCES}

Andrews, D. G., 1983: A finite-amplitude Eliassen-Palm theorem in isentropic coordinates. J. Atmos. Sci., 40, 1877-1883, doi:10.1175/1520-0469(1983)040<1877:AFAEPT>2.0.CO;2.

_ vertical shear: the generalized Eliassen-Palm relation and the mean zonal acceleration. J. Atmos. Sci., 33, 2031-2048, doi:10.1175/1520-0469(1976)033<2031:PWIHAV>2.0.CO;2.

Butchart, N., and E. Remsberg, 1986: The area of the stratospheric vortex as a diagnostic for tracer transport on an isentropic surface. J. Atmos. Sci., 43, 1319-1339, doi:10.1175/ 1520-0469(1986)043<1319:TAOTSP $>2.0$.CO;2.

Edmon, H., B. Hoskins, and M. McIntyre, 1980: Eliassen-Palm cross sections for the troposphere. J. Atmos. Sci., 37, 2600-2616, doi:10.1175/1520-0469(1980)037<2600:EPCSFT>2.0.CO;2.

Egger, J., and K.-P. Hoinka, 2011: Global angular momentum fluxes in height coordinates. Mon. Wea. Rev., 139, 2552-2560, doi:10.1175/2010MWR3514.1.

Iwasaki, T., 1988: A diagnostic formulation for wave-mean flow interactions and Lagrangian-mean circulation with a hybrid vertical coordinate of pressure and isentropes. J. Meteor. Soc. Japan, 67, 293-311.

- 1992: General circulation diagnosis in the pressureisentrope hybrid vertical coordinate. J. Meteor. Soc. Japan, 70, 673-687.

Johnson, D., 1989: The forcing and maintenance of global monsoonal circulations: An isentropic analysis. Advances in Geophysics, Vol. 31, Academic Press, 43-329, doi:10.1016/ S0065-2687(08)60053-9.

Juckes, M., 2001: A generalization of the transformed Eulerianmean meridional circulation. Quart. J. Roy. Meteor. Soc., 127, 147-160, doi:10.1002/qj.49712757109.

Koh, T.-Y., and R. Plumb, 2004: Isentropic zonal average formalism and the near surface circulation. Quart. J. Roy. Meteor Soc., 130, 1631-1654, doi:10.1256/qj.02.219.

Lorenz, F., 1955: Available potential energy and the maintenance of the general circulation. Tellus, 7, 157-167, doi:10.1111/ j.2153-3490.1955.tb01148.x.

Nakamura, N., 1995: Modified Lagrangian-mean diagnostics of the stratospheric polar vortices. Part I: Formulation and analysis of GFDL SKYHI GCM. J. Atmos. Sci., 52, 2096-2108, doi:10.1175/1520-0469(1995)052<2096:MLMDOT>2.0.CO;2.

__, 1996: Two-dimensional mixing, edge formation, and permeability diagnosed in an area coordinate. J. Atmos. Sci., 53, 1524-1537, doi:10.1175/1520-0469(1996)053<1524: TDMEFA $>2.0 . \mathrm{CO} ; 2$.

Nurser, G., and M.-M. Lee, 2004: Isopycnical avearaging at constant height. Part I: The formulation and a case study. J. Phys. Oceanogr., 34, 2721-2739, doi:10.1175/ JPO2649.1.

Nycander, J., J. Nilsson, K. Döös, and G. Broström, 2007: Thermodynamic analysis of ocean circulation. J. Phys. Oceanogr., 37, 2038-2052, doi:10.1175/JPO3113.1. 
Oort, A., and J. Peixoto, 1983: Global angular momentum and energy balance requirements from observations. Advances in Geophysics, Vol. 25, Academic Press, 355-490, doi:10.1016/ S0065-2687(08)60177-6.

Pauluis, O., and A. Mrowiec, 2013: Isentropic analysis of convective motion. J. Atmos. Sci., 70, 3673-3688, doi:10.1175/ JAS-D-12-0205.1.

Tanaka, D., T. Iwasaki, S. Uno, N. Ujiie, and K. Miyakazi, 2004: Eliassen-Palm flux diagnostics based on isentropic representations. J. Atmos. Sci., 61, 2370-2383, doi:10.1175/ 1520-0469(2004)061<2370:EFDBOI>2.0.CO;2.
Tung, K. K., 1986: Nongeostrophic theory of zonally averaged circulation. Part I: Formulation. J. Atmos. Sci., 43, 2600-2618, doi:10.1175/1520-0469(1986)043<2600:NTOZAC >2.0.CO;2.

Yang, H., K. K. Tung, and E. Olaguer, 1990: Nongeostrophic theory of zonally averaged circulation. Part II: EliassenPalm divergence and isentropic mixing coefficient. J. Atmos. Sci., 47, 215-241, doi:10.1175/1520-0469(1990)047<0215: NTOZAC $>2.0 . \mathrm{CO} ; 2$.

Zdunkowski, W., and A. Bott, 2003: Dynamics of the Atmosphere: A Course in Theoretical Meteorology. Cambridge University Press, 738 pp. 